\title{
A COMPARISON OF FLASH ELECTRORETINOGRAMS RECORDED FROM BURIAN ALLEN, JET, C-GLIDE, GOLD FOIL, DTL AND SKIN ELECTRODES
}

\author{
L. ESAKOWITZ, A. KRISS and F. SHAWKAT \\ London
}

\begin{abstract}
SUMMARY
Single flash scotopic and photopic electroretinograms (ERGs) were recorded from the same subjects using six types of corneal electrode, in order to assess their relative effectiveness. In addition, the ERG from a lower eyelid skin electrode was recorded to give an indication of the degree of attenuation to be expected from a skin electrode. On average, the scotopic ERG recorded from the Burian Allen electrode measured $471 \mu \mathrm{V}(\mathbf{1 0 0 \%})$, and relative to this the b-waves recorded using other electrodes were as follows: JET (89\%), C-glide (77\%), gold foil $(56 \%)$, DTL $(46 \%)$ and skin (12\%). Under photopic conditions the order was the same and the interelectrode proportions similar. The b-wave amplitude recorded using the Burian Allen electrode was $125 \mu \mathrm{V}(100 \%)$, and with other electrodes was as follows: JET $(93 \%)$, C-glide $(78 \%)$, gold foil $(60 \%)$, DTL $(60 \%)$ and skin $(14 \%)$.
\end{abstract}

There are many types of electroretinogram (ERG) electrode commercially available; however, there appears to be little information comparing their recording efficacy, practicalities of insertion and comfort of wear. Contact lens electrodes are advocated when recording single flash ERGs from adults. ${ }^{1}$ Lightweight, lower lid, hook electrodes and thread electrodes which contact the cornea and do not interfere with the optics of the eye are commonly chosen for recording the pattern ERG, and some workers use them also for recording the single flash ERG to ganzfeld stimulation. ${ }^{2}$ Neither contact lens nor lightweight eye contact electrodes are tolerated by most young children. Some laboratories resort to recording contact lens ERGs while the child is conscious and if necessary restrained, ${ }^{3,4}$ or unconscious while under the influence of a sedative or general anaesthetic. Other centres use electroencephalogram (EEG) skin electrodes placed under the eye or on the bridge of the nose, and record an averaged ERG from conscious children (often the visual evoked potential is concurrently recorded also). ${ }^{5,6}$

Correspondence to: Dr. A. Kriss, Eye Department, Hospital for Sick Children, Great Ormond Street, London WC1N 3JH, UK.
We have recorded scotopic and photopic ERGs using six types of corneal electrode in order to assess their relative efficacy and to provide a rational basis for selecting between them. In addition, the ERG was concurrently recorded from the lower eyelid to give an indication of the degree of attenuation to be expected from skin electrodes.

\section{METHODS}

ERG recordings were made from 4 young healthy adults. The test procedure was explained to subjects and all consented to participation in the study. Pupils were fully dilated with $1 \%$ tropicamide, and $1 \%$ amethocaine was used to induce topical anaesthesia prior to application of corneal electrodes. Each subject was tested with the following electrodes: the Burian Allen unipolar contact lens electrode with lid speculum, the JET disposable contact lens electrode, the C-glide carbon fibre electrode, the gold foil and the DTL silver-impregnated nylon thread electrode (Fig. 1). In addition, a silver/silver chloride EEG electrode was attached centrally below the pupil; the centre of the electrode was $1 \mathrm{~cm}$ below the lower eyelid margin. A mid-frontal EEG electrode (Fz, 10-20 system) served as common reference for all the recordings. Each electrode was kept in place for the duration of the test, a period of approximately 30 minutes. The testing order of electrodes varied between subjects.

A Grass photic stimulator (Model PS 22) was used to produce white flashes (intensity $4,4.75 \times 10^{6}$ lumens). The lamp had a diffusing screen and was placed in close proximity to the subject, $20 \mathrm{~cm}$ from the tested eye. Single flashes were recorded on a Medelec Sensor averager. The amplifier bandpass was set between 1 and $60 \mathrm{~Hz}$. For each electrode two single ERGs were recorded under photopic conditions. Following a period of 15 minutes in complete darkness, two single flash ERGs were recorded with a separation of 1 minute. All subjects were tested between 10 a.m. and 1 p.m.

\section{RESULTS}

Clear, consistent differences were found when comparing 


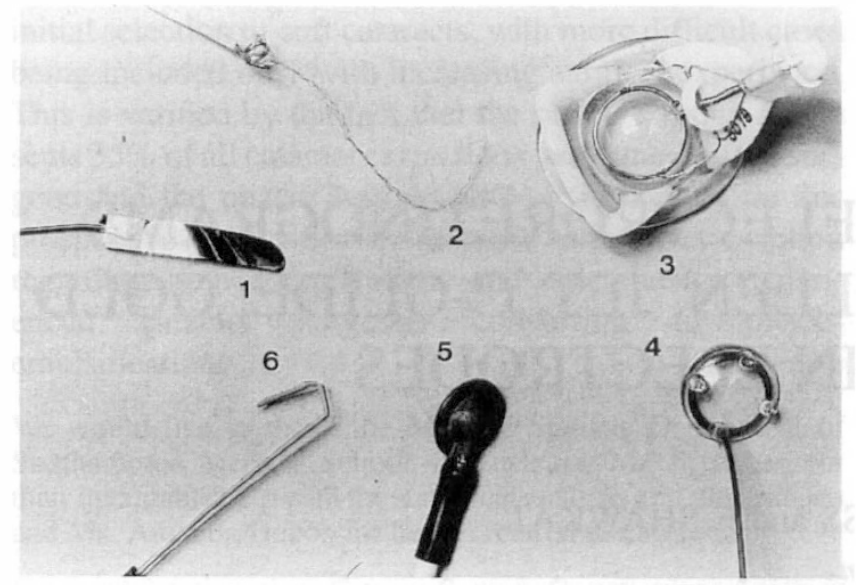

Fig. 1. Electrodes tested: 1, gold foil; 2,DTL; 3, Burian Allen; 4, JET; 5, EEG skin; 6, C-glide.

the amplitude of ERGs recorded from the corneal electrodes. Figs. 2 and 3 give the mean b-wave amplitude and standard deviation for scotopic and photopic conditions, respectively. Independent statistical analysis for both scotopic and photopic data using Friedman two-way analysis of variance of ranks showed a significant trend $(p<0.02)$. In both conditions the peak-to-peak amplitude of the b-wave was largest for the Burian Allen electrode, followed in descending order by the JET, C-glide, gold foil and DTL electrodes. On average, the Burian Allen scotopic b-wave measured $471 \mu \mathrm{V}$ (100\%), and relative to this the amplitudes from the other electrodes were as follows: JET (89\%), C-glide (77\%), gold foil (56\%), DTL $(46 \%)$ and skin (12\%) (Fig. 2). Under photopic conditions the relative proportions were in the same order: the Burian Allen photopic b-wave amplitude measured $125 \mu \mathrm{V}$ (100\%), and for the other electrodes as follows: JET (93\%), C-glide (78\%), gold foil (60\%), DTL (53\%) and skin (14\%) (Fig. 3). There were no significant differences in b-wave latency when comparing electrodes.

\section{DISCUSSION}

The results from the 4 subjects were consistent and clearly

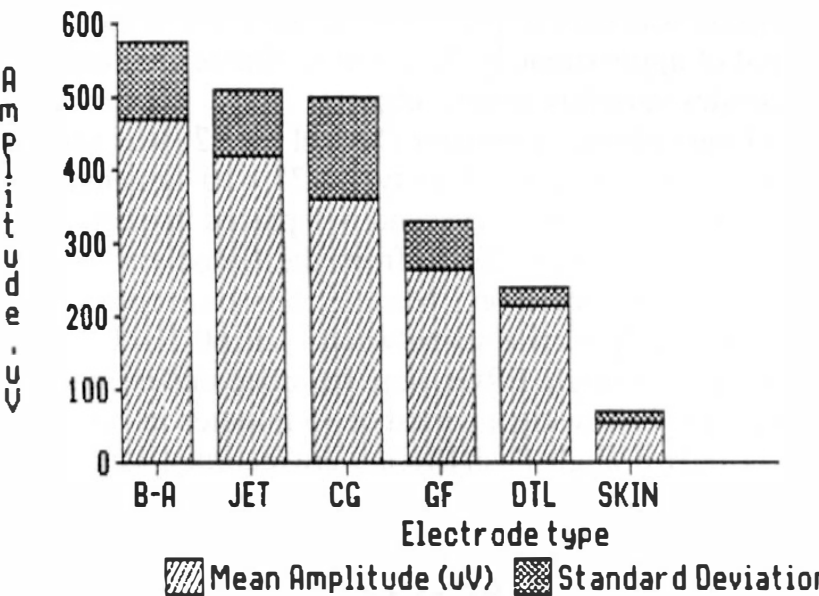

Fig. 2. Scotopic condition. Mean amplitude and standard deviation for single flash and averaged ERGs from the different electrodes.

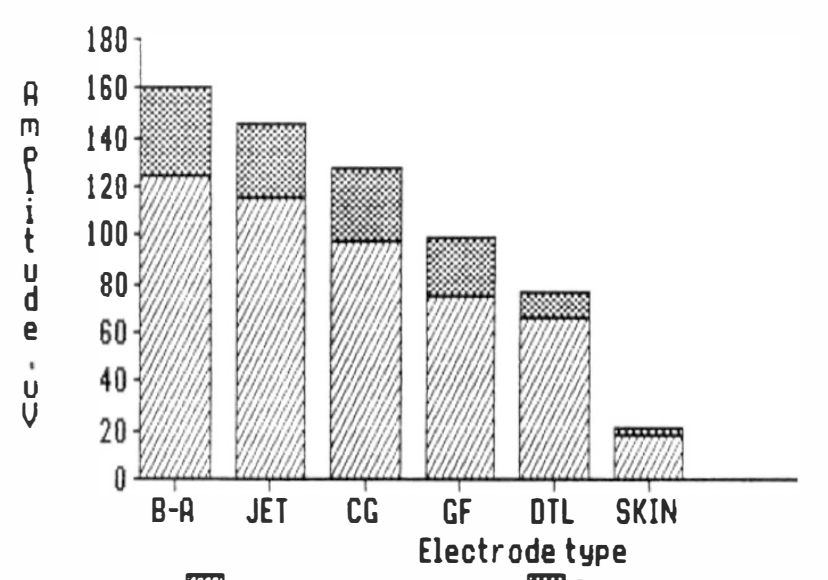

[D] Mean Amplitude(uV) 䍑 St andard Deviation

Fig. 3. Photopic condition. Mean amplitude and standard deviation for single flash and averaged ERGs from the different electrodes.

demonstrate that the contact lens electrodes give larger ERGs compared with gold foil, carbon fibre (C-glide) and nylon fibre (DTL) electrodes (Fig. 4). It is likely that these differences are due to physical factors governing the amount of current flow through electrodes, such as the resistive and capacitative properties of materials from which they are made and are coated with, and the area of the recording surface. ${ }^{7}$ In particular, the DTL electrode had a DC resistance of $170 \mathrm{k} \Omega$, which is about 10 times that of other corneal electrodes. Spontaneous movements of the eye relative to the recording electrode may also lead to differences in ERG size. This effect will be greatest for lower lid hook electrodes which have a fixed position and smaller areas of contact compared with lens electrodes.

Our results are consistent with those of other studies

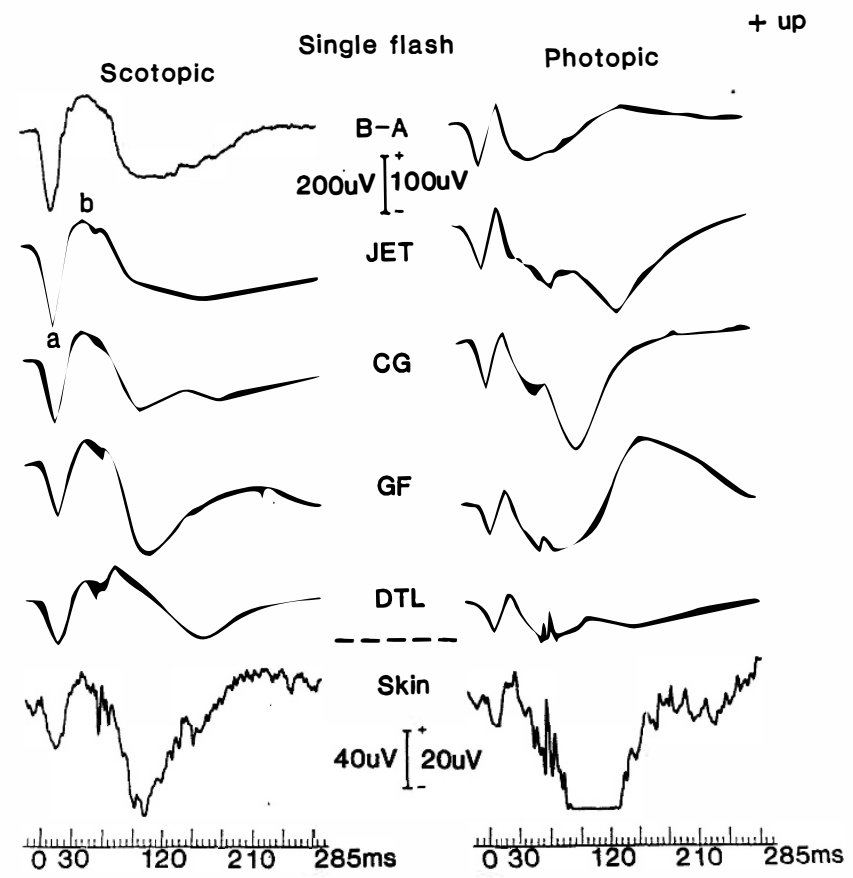

Fig. 4. Group average ERGs from 4 subjects. Single flash ERGs recorded under scotopic (left) and photopic (right) conditions. 
comparing ERG electrodes. Most studies agree that contact lens electrodes give the largest ERGs, though there is some degree of discrepancy in the relative size of ERGs recorded from non-contact-lens electrodes. The order of our results agrees closely with those of Robbins and Turner, ${ }^{8}$ who used the Henkes contact lens electrode and found it gave the largest scotopic b-wave $(525 \mu \mathrm{V}=$ $100 \%)$ compared with JET $(76 \%)$, C-glide $(67 \%)$, gold foil (57\%), DTL (19\%) and skin (10\%). Gjotterberg ${ }^{9}$ darkadapted subjects for 30 minutes and found that the Lovac vacuum electrode gave larger ERGs (scotopic b-wave = $445 \mu \mathrm{V}=100 \%)$ compared with the JET (79\%), Burian Allen (75\%) and gold foil (57\%). However, others have found non-contact-lens electrodes to give relatively larger ERGs; Dawson et al.$^{10}$ reported that scotopic ERGs from DTL electrodes are only about $10 \%$ smaller than ERGs from contact lens electrodes (Burian Allen or Henkes), and Arden and colleagues ${ }^{2}$ showed that the scotopic ERGs from gold foil electrodes are about $25 \%$ smaller than ERGs from Karpe lens electrodes. We found that the ERG from lower lid skin electrodes was of a similar morphology and latency to Burian Allen recordings; however, ERGs were $86-88 \%$ smaller and somewhat noisier due mainly to intrusion of electromyographic activity. Averaging the single flashes greatly helps to improve the signal-to-noise ratio. Coupland and Janaky ${ }^{11}$ found scotopic skin ERGs were between $43 \%$ and $73 \%$ the size of those recorded from DTL electrodes.

We also found that there were differences between electrodes when considering ease of insertion and comfort during wear. None of the electrodes tested could be considered as ideal. The Burian Allen was not always easy to insert, particularly if the palpebral fissure was narrow. Some subjects found it restrictive and uncomfortable during wear. The JET electrode was more comfortable and easier to put in place than the Burian Allen; however, it was readily displaced if the subject sat upright. The coarse recording tip of the $\mathrm{C}$-glide electrode and the pre-shaped plastic hook which inevitably touched the eyelashes, made it irksome and the most unpopular of the eye contact electrodes. The gold foil electrode was well tolerated and relatively simple to insert, though in some subjects it was repeatedly displaced by the eyelashes during blinking; also this electrode is pliable and the forming of an arc over the lower eyelashes requires practice and dexterity. All the subjects considered the DTL electrode to be the most comfortable, but compared with the other electrodes its light, fine, multi-threaded structure made it awkward to place along the rim of the lower eyelid. Skin electrodes were very easy to apply, though scarification of the underlying skin was often needed to ensure a low impedance contact and this was considered uncomfortable by most subjects.

We thank the Help a Child to See charity and The Wooden Spoon Society for their financial support. Mr. Leonard Esakowitz was supported by the Grampian Health Board.

Key words: Electroretinogram, Electroretinogram electrodes.

\section{REFERENCES}

1. Marmor MF. Standard for clinical electroretinography: International Standardisation Committee. Arch Ophthalmol 1989;107:816-9.

2. Arden GB, Carter RM, Hogg C, Siegel IM, Margolis S. A gold foil electrode: extending the horizons for clinical electroretinography. Invest Ophthalmol Vis Sci 1979;18:421-6.

3. Marmor MF. Corneal electroretinograms in children without sedation. J Pediatr Ophthalmol 1976;13:112-8.

4. Fulton AB, Hartmann EE, Hansen RM. Electrophysiologic testing techniques for children. Doc Ophthalmol 1989;71: 341-7.

5. Harden A, Adams GG, Taylor DS. The electroretinogram. Arch Dis Child 1989;64:1080-7.

6. Kriss A, Russell-Eggitt I. Electrophysiological assessment of visual function in infants. Eye 1992;6:145-53.

7. Geddes LA. Electrodes and the measurement of bioelectric events. New York: Wiley, 1972.

8. Robbins J, Turner J. Assessment of various types of electrodes in clinical ERG. Impulse 1988;5:2-5,12 (Medelec International in-house publication).

9. Gjotterberg M. Electrodes for electroretinography: a comparison of four different types. Arch Ophthalmol 1986;104: 569-70.

10. Dawson WW, Trick GL, Maida TM. Evaluation of the DTL corneal electrode. Doc Ophthalmol 1982;31:81-8.

11. Coupland SG, Janaky M. ERG electrode in pediatric patients: comparison of DTL fibre, PVA-gel, and non-corneal skin electrodes. Doc Ophthalmol 1989;71:427-34. 